\title{
EFFECTIVE FINITE ELEMENT SOLUTION AND POST-PROCESSING FOR WIDE LOAD SPECTRUM
}

This paper presents a computational algorithm allowing the efficient use of high-performance computing resources to solve nonlinear problems of continuum mechanics using FEM. The algorithm is suitable for solving static problems and tasks of estimating lifetime of structures in cases where the load is defined by the load spectrum. The complexity of the problem increases significantly in the case of nonlinear problems. The principle of the stress superposition cannot be used in that case. For each loading mode a separate FE analysis must be made.

A quick and efficient procedure for evaluating the results of mentioned analysis for the static calculation as well as for the estimated life calculation is also presented in this paper. In the presented examples the contact of bodies is used as a source of nonlinearity. The size, shape or position of the contact area is not possible to estimate in contact of bodies. These types of problems are thus highly nonlinear. Programs for the presented algorithms are processed in the program package OCTAVE and calculations using FEM are made in the software ADINA.

Keywords: FEM, static analysis, lifetime, spectrum of load, contact of bodies, state of stress, stress tensor, finite element mesh, ADINA, OCTAVE.

\section{Introduction}

Finite element method (FEM) is most commonly used in continuum mechanics for the analysis of stress state. Together with the increasing computing power, the complexity of the tasks also increases. The preparation of necessary matrices and their solution takes currently from several minutes to several tens of hours. The fact is that the user is willing to accept such a solution time and is ready to lower requirements for the accuracy to speed up the solution.

For static tasks solution it is mostly necessary to analyze stress state for a relatively small number of load spectrum (about tens of load states - lines of the load spectrum). This load spectrum is defined by a small group of phenomena which cause extreme loads during operation. Degree of safety against yield strength and strength limit of the used material is checked in the analysis. The aim of the static analysis is to ensure the operational safety and to prevent the sudden loss of the construction utility properties (change in shape, brittle fracture, etc.) [1].

Solving the estimated life of the structure requires an analysis of the state of stress for the given load spectrum which has a relatively low number of loads (force, moment, pressure) and a relatively high number of loading states. Such a load spectrum is usually defined by the time course of the dynamic load and it may have the character of accidental load.
Methods for reducing the number of load steps are often used to solve this kind of load spectrum. The final load spectrum may still contain tens to thousands of different loading states [2, 3, 4 and 5].

The main criterion for the establishment of an efficient algorithm for solving the above mentioned tasks is the relationship between the load and the resulting state of stress. This relationship can be linear or nonlinear.

\section{Algorithm for linear relationship between load and state of stress}

The principle of stress superposition can be used to speed up analysis of the state of stress in the case of linear relationship. Algorithm based on the principle of stress superposition is defined as follows:

1. Determine the number of individual loads (forces, moments, pressures). Only one single load is always considered.

2. FE analysis is performed for each individual load. The load size is modified so that the load will be unitary. The absolute size of all the loads shall be taken into account for normalization of loads. The unitary load is chosen in multiples of basic units.

\footnotetext{
* Marian Handrik, Milan Vasko, Peter Kopas, Milan Saga

Department of Applied Mechanics, Faculty of Mechanical Engineering, University of Zilina, Slovakia

E-mail: marian.handrik@fstroj.uniza.sk
} 
3. Analysis of the stress state for all types of loads and listing of the stress tensor components for all nodes of finite element mesh.

4. Calculation of the stress state for individual load cases (lines of the spectrum of load). The stress tensor is multiplied by a load coefficient at each point of the finite element mesh for the relevant unitary load. The individual components of stress tensors are then summed together because in the calculation all loads for the corresponding load state shall be included.

5. The processing of the analysis results for individual lines of the load spectrum, determination of the static safety level and calculating the estimated life of the structure.

It is necessary to implement $N$ independent analyses of the state of stress if the $N$ individual loads (forces, moments, and pressures) are defined in the spectrum. Arrays of stress tensors are subsequently written to the files. For each load a single file is obtained. This file contains six columns $\left(\sigma_{x x}, \sigma_{y y}, \sigma_{z z}, \sigma_{x y}, \sigma_{x z}, \sigma_{y z}\right)$ and the number of rows corresponds to the number of nodes in finite element mesh. These files are read and used for calculation of the stress state (using the method of stress superposition [6].

In general, analysis of the stress state using the FEM is significantly more time consuming than reading the arrays of stress tensors and their multiplication and addition. The proposed algorithm allows a significant reduction in the time required for analysis of an entire spectrum of loads. The procedure can be easily parallelized in the phase of stress state analysis by FEM. Individual analyses may be solved on separate computers [7].

\section{Algorithm for nonlinear relationship between load and state of stress}

The principle of superposition of stress cannot be used in nonlinear relationship between the load and the resultant array tensors of stress at nodes of finite element mesh. A suitable example of such a nonlinear relationship is the contact of bodies. Contact of bodies is highly nonlinear problem with regard to the previously unknown size, shape or position of the contact area. For the solution of the contact analysis the significantly higher number of steps in nonlinear analysis is needed than for solving geometric and material nonlinearities.

The contact of bodies between several parts of the structure is defined in the example below. The bolts with a defined preload are used in this example. In the first step of the contact problem solution (time $t=0$ ) it is necessary to eliminate the initial penetrations of the contact bodies. The initial preload in the bolds needs to be solved at the same time.

Powerful computing resources in the form of a cluster enable parallelization of the computing algorithms. The cluster has 46 computational nodes (each computational node has 12 cores and 48 gigabytes of RAM). The algorithm for solution of nonlinear problems with the contact of bodies is as follows:
1. Preparation of the model and generating files needed for the analysis of a contact at time $t=0$. Saving the model with finite element mesh in a binary form. The initial penetrations of the contacts of bodies are eliminated in this introductory calculation. Next, the shortening of bolts is calculated to apply the prescribed force acting in them. Analysis of the stress state is solved for the unitary loads at time $t=1$. This analysis is needed for the next restart of the analysis at the same time. A separate time function is defined for each load.

2. Generating input files for individual calculations corresponding to different load states. Stored model is loaded in a binary form with the finite element mesh in the phase of generating. Restart of the analysis is defined for time $\mathrm{t}=1$. Time functions for individual loads are adjusted to correspond to the actual loads for individual load states.

3. The inclusion of tasks to the queue for middleware TORQUE which manages the calculations on the grid. Files needed to calculate the individual tasks are generated. Analysis of the state of stress by FEM is made. Analysis of the results and listing the individual components of stress tensor at all nodes of finite element mesh is made.

4. Checking if all tasks from the queue of managing system on the grid are processed. The monitoring of the completion of all tasks is performed periodically and the continuation is possible only after completion of all the tasks.

5. The processing of the analysis results for individual lines of the load spectrum, determination of the static safety level and calculating the prediction of lifetime of the structure.

\section{Analysis of the results of static task for the spectrum of load}

State of stress in the structure is most often evaluated for static analysis. Analysis of the results consists of creating the graphs of stress area distribution where points with increased values of stress are evaluated. The position of these points and corresponding numeric value are determined in this evaluation. The evaluation must be made for all analyzed states of load; therefore, it must be performed across the whole spectrum of load. Then, it is necessary to make the resulting evaluation of the state of stress.

Points of stress concentration may be located in several areas of the construction and results may correspond to various loading states. Therefore, each load state has its own graphical outputs where stress concentrations are to be displayed and located. This procedure becomes time consuming with a greater number of loading states. Its difficulty is based mainly on mutual comparison of the points and values of stress (and corresponding numeric arrays) on different graphs.

Both above mentioned algorithms for the analysis of stress state are designed to allow the use of cluster parallelization. The 
results of the state of stress analysis for individual load cases are stored in a text file and they are ready for further processing. The algorithm for processing the results of static analysis in which the resulting state of stress and equivalent stress according to the required criteria (von Mises, Tresca) at each point of finite element mesh is evaluated as follows:

1. Processing of the results of analyses for individual load cases. The stress tensor for individual nodes of the finite element mesh is written to a separate file. All equivalent stresses determined in accordance with required criteria will be recorded into the second separate file. Maximum of 6 values can be written into one file. If necessary, several files with the results must be created (these files can be created immediately after analysis of the state of stress). Within the cluster solution, parallelization of the algorithm for stress state analysis is used.

2. The program package OCTAVE reads saved files in successive steps corresponding to the different states of load. The maximum values for individual components of the stress tensor from previous loading states and those from current evaluation are compared. The serial number of the load state which caused the maximum value of the corresponding element of the stress tensor is also saved.

3. The results of the required criteria for evaluation of the equivalent stresses are processed. The serial number of the load state which caused the given state of stress is saved too.

4. Generating an input file for post-processing. It allows viewing the cumulative results and creation of graphical outputs from these results.

Equivalent stresses must always be evaluated for the individual states of load. The realization is most suitable in the program for the analysis of stress state using FEM - in our case - software ADINA. The equivalent stresses cannot be calculated from the cumulative stress tensor. This stress tensor contains the maximum values of stresses of the entire spectrum of loads. The individual

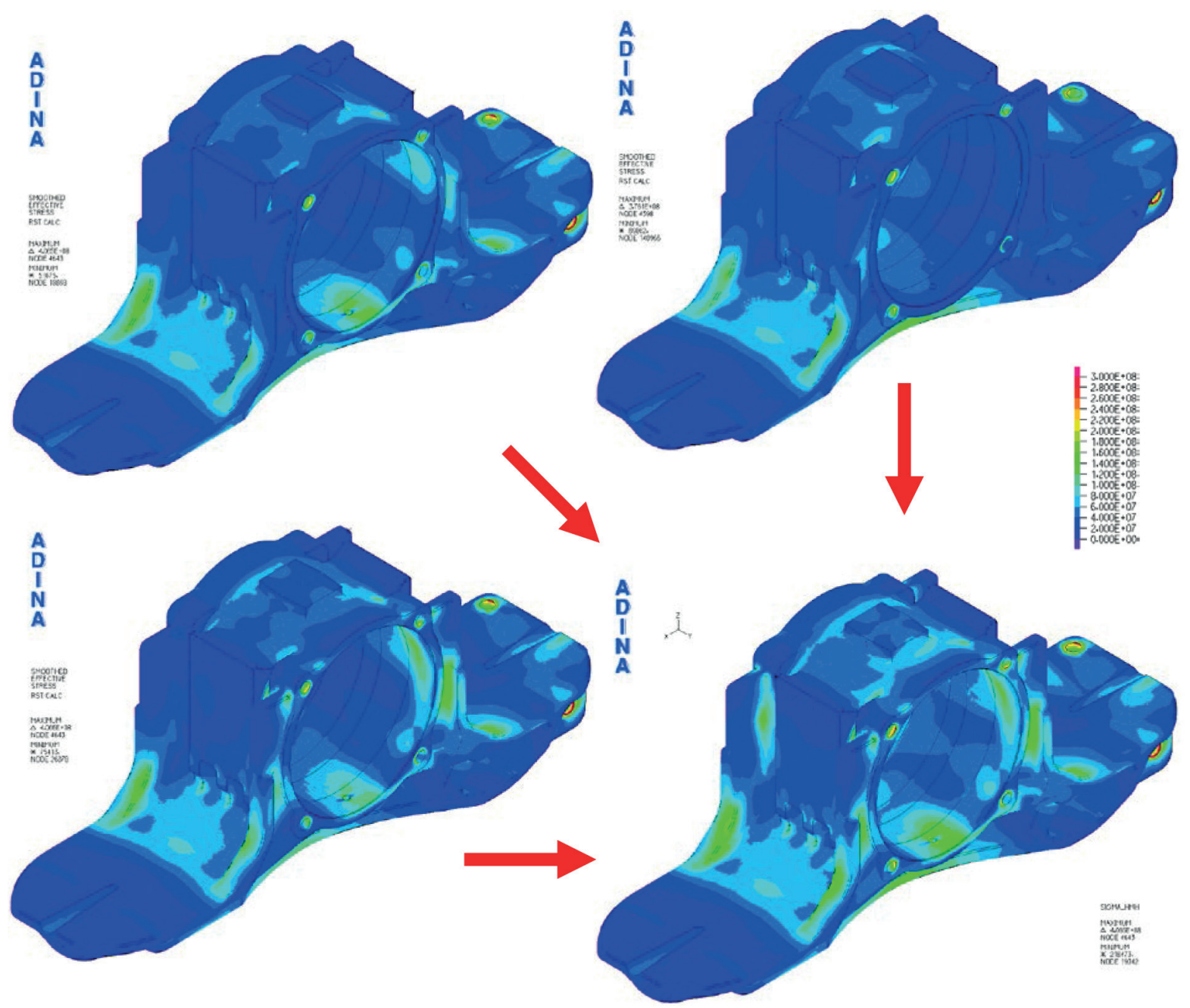

Fig. 1 Cumulative effective stress distribution results [Pa] 
tensor components may, however, correspond to the different load cases [8].

The above mentioned processing of the state of stress analysis for the individual load cases allows a simplified evaluation of the results for the entire load spectrum. The maximum values of the corresponding stress tensor component or equivalent stress processed for the entire load spectrum can be displayed in one figure (Fig. 1). Registered serial number of the load state that achieved a maximum value of the relevant component of stress tensor or the equivalent stress allows a simple graphic representation of the corresponding state. The practical experience with this procedure for the model about the size of 1.2 million nodes shows that the reading and processing of one load stave with two sets of results takes approximately 80 seconds

\section{The review of methods for the fatigue life evaluation of structural elements}

Most of the criteria for the calculation of fatigue life used to estimate the life of the structure use knowledge of the stress state or knowledge of the deformation in the structure. The methodologies based on the evaluation of the extreme state of load are used to calculate the fatigue life of machine parts. In the first case, they evaluate the mode which causes maximum stress or strain [5 and 9]. In the second case cumulative criteria are used where each state of load contributes to the total damage. Using referred methodologies with regard to their specific state of load it is possible to predict the number of cycles to fracture $N_{f}$ [10 and 11]

Input for the analysis of the fatigue life is the geometry, load and material properties of components or structures. Strength and fatigue properties (Wohler or Manson-Coffin curve) of the material are most commonly determined from the database or from the experiment. Loads can be obtained by experiment, analytical calculation, by estimation or based on the use of numerical methods. Based on these data it is possible to calculate the local stresses [12 and 13]. In the first case, the input for the analysis of lifetime is the file with a set of stresses for each load state and the file containing the corresponding loading process. In the second case, the file contains stresses for each load state and the number of load cases corresponds to the number of eigenvalue shapes of the flexible element used in the calculation. Loading history is analogously assigned to each mode. The output of the analysis is the lifetime calculation for each node of components or structure [14].

The main methods for predicting the fatigue life of the structures are based on:

the nominal stresses,

the local elastic stresses,

the local elastic-plastic stresses and deformations,

the fracture mechanics.
The S-N (Stress-Life) method and NSA (Nominal Stress Analysis) method belong to lifetime estimation methods which are based on the nominal stresses. Determination of fatigue life based on the S-N curve is inaccurate especially in low cycle fatigue. It is the oldest method which can be used for a wide range of applications. The method provides an extensive database and its use is appropriate for the high cycle fatigue. The NSA method uses the relationship between the size of the nominal stress of cycles and the number of cycles to failure. The method uses correction of medium stress according to Goodman or Gerbera.

The LESA (Local Elastic Stress Analysis) method belongs to lifetime estimation methods which are based on the local elastic stress. The method uses the relationship between the size of the local elastic stress of cycles and the number of cycles to failure. This method is a modification of the NSA method. The method is particularly useful in high cycle fatigue and uses the same corrections of medium stress as the NSA method.

The $\varepsilon-\mathrm{N}$ (Strain-Life) method and LPSA (Local Plastic Strains and Stress) method belong to lifetime estimation methods based on the local elastic-plastic stresses and deformations. The LPSA method is based on the testing of the material in the context of local deformation according to cycles in order to initiate macroscopic cracks. This approach allows the analysis of elastic-plastic deformation at the critical points. The method uses (in uniaxial stress) mostly values of the stresses and strains identified on the basis of elastic calculation. The method subsequently makes the elastic-plastic correction according to the most common methods of Neubert, Mertens-Dittmann or SeegerBest. Methods of SWT (Smith, Watson, Topper) or Morrow most frequently used for medium stress corrections. The values of the local elastic-plastic stresses and strains can also be determined using a nonlinear elastic-plastic calculation. In this case it is necessary to make a separate calculation for each local maximum of the load. This is time consuming and puts considerable demands on the computing power.

The FMA (Fracture Mechanics Analysis) method belongs to lifetime estimation methods that are based on fracture mechanics. The linear-elastic fracture mechanics analysis most often uses the Paris rule for predicting the crack extension. The size of crack increase and stress intensity factor at the end of the crack is brought into relationship. The method is mostly used in the aerospace industry for the calculation of the allowable damage (Damage-Tolerant). The calculation allows reliable monitoring of the crack of a certain size. This approach enables monitoring of crack growth in each step - cycle after cycle.

In addition to these most commonly used methods of fatigue life evaluation, there are also other methods for calculating the number of cycles to failure of machine parts [15]. This group of methods includes the following methods - Vibration Fatigue Analysis, Biaxial Fatigue Analysis, Multiaxial Fatigue Analysis, Global Multiaxial Fatigue (Safety Factor Analysis, Dang Van, McDiarmid, Crack Initiation Life Analysis, SWT- 
Bannantine, Fatemi-Socie, Wang-Brown, Normal Strain, Shear Strain), Local Multiaxial Fatigue (Crack Initiation Life Analysis, Mròz-Garud, Wang-Brown criterion, Multiaxial Elasto-plastic Neuber Correction), Welds Fatigue Analysis and Temperature Dependent Fatigue etc.

\section{Analysis of the results for the dynamic load spectrum and lifetime rating of the structure with respect to the Smith's diagram}

Evaluation of fatigue life based on a state of stress evaluation in the construction with respect to the Smith diagram determines if the state of stress is in the stress scope which causes a critical accumulation of fatigue damage. For the lifetime evaluation of the structure with respect to the Smith diagram, the value of average stress and amplitude of the stress range is necessary to know [4, 5 and 9].

Algorithm for lifetime evaluation of the structure with respect to the Smith diagram has the following form:

1. The first analysis of the results is made in post-processing of the FEM software ADINA; the principal stress $\sigma_{1}$ and directional angles of the first principal stress are calculated. The results are written to a text file. Equivalent stresses according to the von Mises method will also be written to the file.

2. The second analysis of the results is made in post-processing of the FEM software ADINA; the individual components of stress tensor are displayed. Both analyses of results are made one after the other within the results loading in postprocessing.

3. Files containing the principal stress $\sigma_{1}$ and directional angles of the first principal stress are sequentially loaded in the program package OCTAVE [16]. After loading the first principal stress for one load state, the result is compared with the maximum value in the previous states. The maximum value of the first principal stress, its directional vectors and the serial number of the load state will be found. The maximum tensile stress $\left(\sigma_{\max }\right)$ of the entire spectrum of the dynamic load on each point of finite element mesh is determined in this step (Fig. 2).

4. The stress tensor for individual load states is sequentially loaded after the determination of the maximum first principal stress and its direction. The stress tensor is projected into the direction of the main stress according to the equation

$$
\mathrm{p}=\mathrm{T}_{\sigma} \cdot \mathrm{n}=\left[\begin{array}{lll}
\sigma_{x} & \tau_{x y} & \tau_{x z} \\
\tau_{y x} & \sigma_{y} & \tau_{y z} \\
\tau_{z x} & \tau_{z y} & \sigma_{z}
\end{array}\right] \cdot\left\{\begin{array}{c}
\cos (\alpha) \\
\cos (\beta) \\
\cos (\gamma)
\end{array}\right\}
$$

From the entire spectrum of the load the minimum value of the stresses in the direction of the maximum principal stress is found. In this step, the minimum tensile stress $\left(\sigma_{\min }\right)$ or maximum pressure stress of the entire spectrum of the dynamic load is determined (Fig. 3).

5. The values of average stress and amplitude of the stress range are determined from values $\sigma_{\min }$ and $\sigma_{\max }$ as follows (Fig. 4 and 5):

$$
\sigma_{m}=\frac{\sigma_{\max }+\sigma_{\min }}{2} \text { and } \sigma_{a}=\frac{\sigma_{\max }-\sigma_{\min }}{2}
$$

6. Evaluation of the degree of safety against the fatigue damage of the structure with respect to the Smith's diagram (Fig. 6) is carried out as follows - for the real value of average stress determined by the relation in point 5 - the maximum allowable value of the stress amplitude $\left(\sigma_{a L I M}\right)$ is subtracted in the Smith's diagram. The safety coefficients are subsequently determined according to the relation (Fig. 7 and 8)

$k=\frac{\sigma_{a L I M}}{\sigma_{a}}$.

7. The processing of equivalent stresses according to von Mises method is identical to the static loading mode.

8. The input for the post-processing is generated. The file allows display of the cumulative results and creation of their graphical outputs.

The principal stresses calculation is made within the postprocessing in the program ADINA. This procedure is faster than the calculation in the program OCTAVE. Both post-processing steps in $1^{\text {st }}$ and $2^{\text {nd }}$ point of the above mentioned algorithm are performed during one loading of results files into the program ADINA. This part can be repeated in a parallel mode immediately after the analysis of stress state.

The practical experience with this procedure (for the model about the size of 1.2 million nodes) shows that the reading and processing of one load state with two sets of results takes approximately 250 seconds.

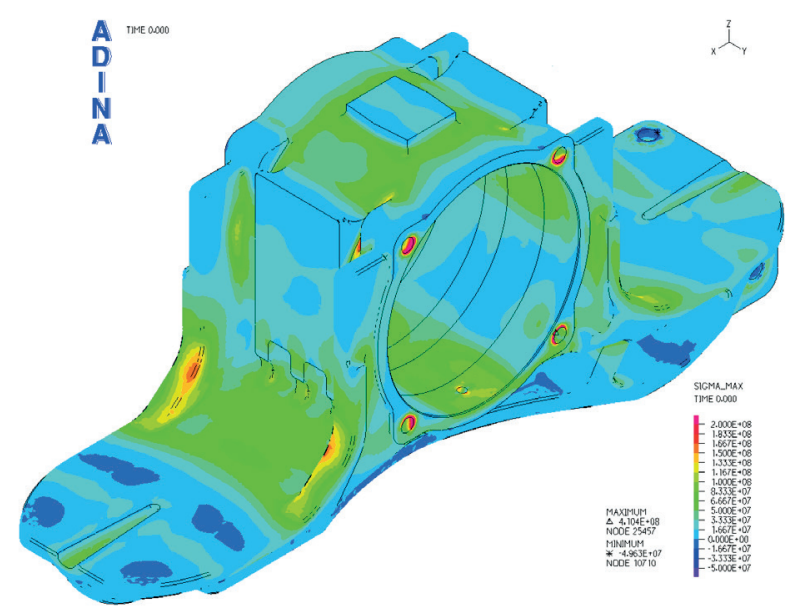

Fig. 2 The maximum tensile stress $\sigma_{\max }[\mathrm{Pa}]$ 


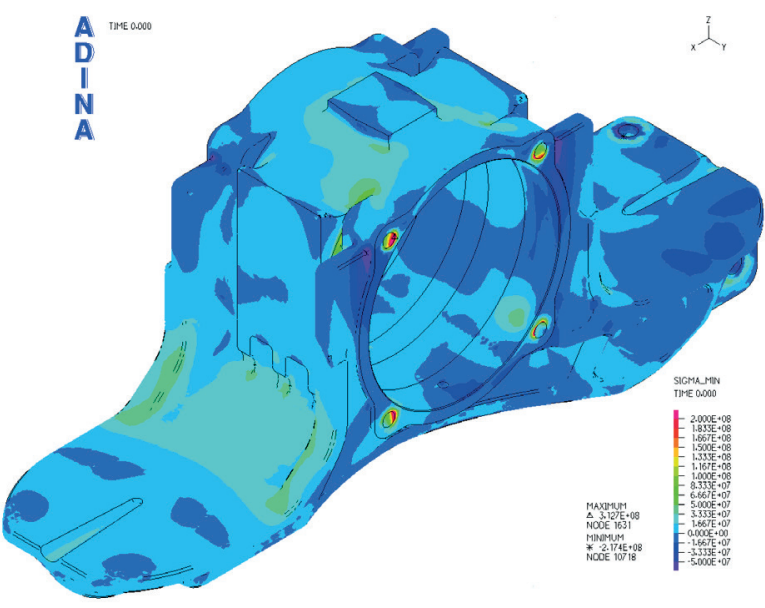

Fig. 3 The minimum tensile stress $\sigma_{\text {min }}[\mathrm{Pa}]$

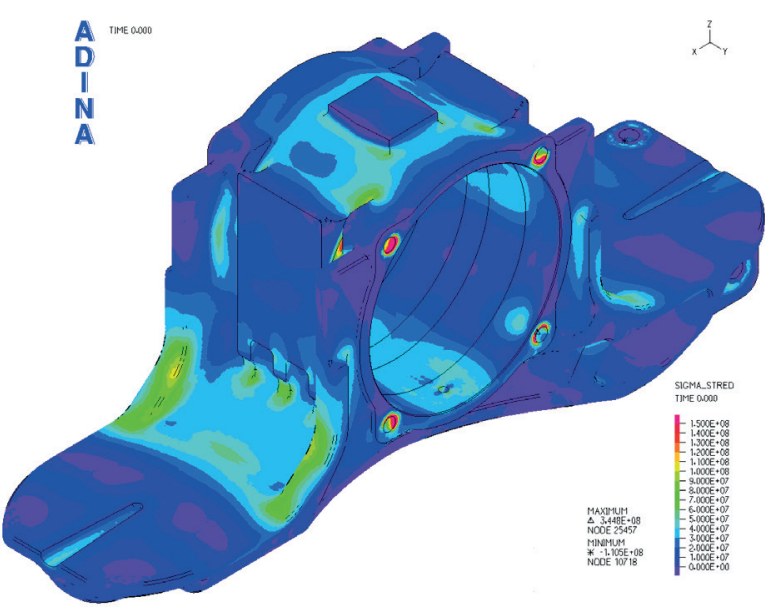

Fig. 4 The average stress $\sigma_{m}[\mathrm{~Pa}]$

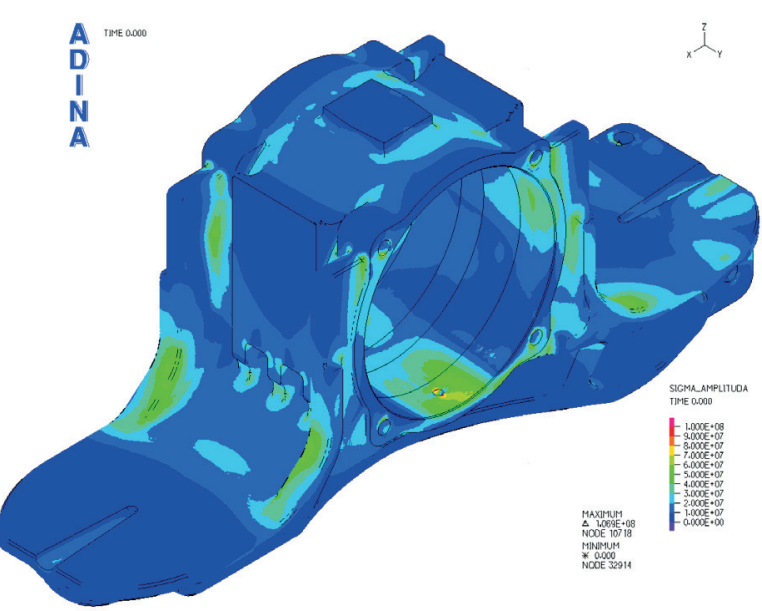

Fig. 5 The amplitude of the stress range $\sigma_{a}[\mathrm{~Pa}]$

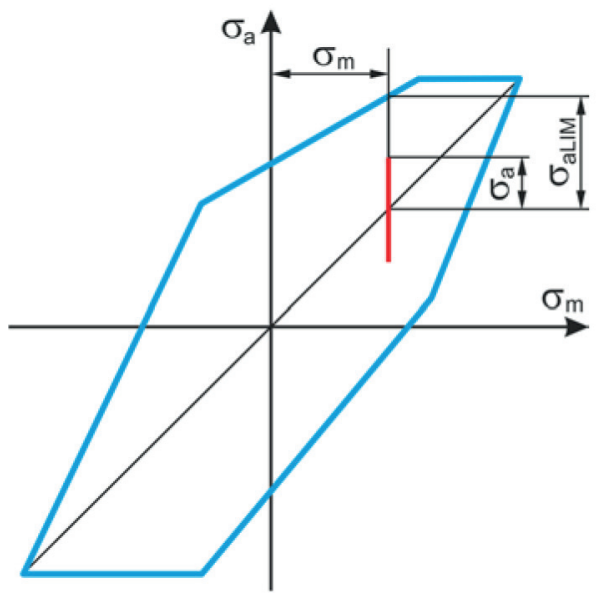

Fig. 6 Smith's diagram

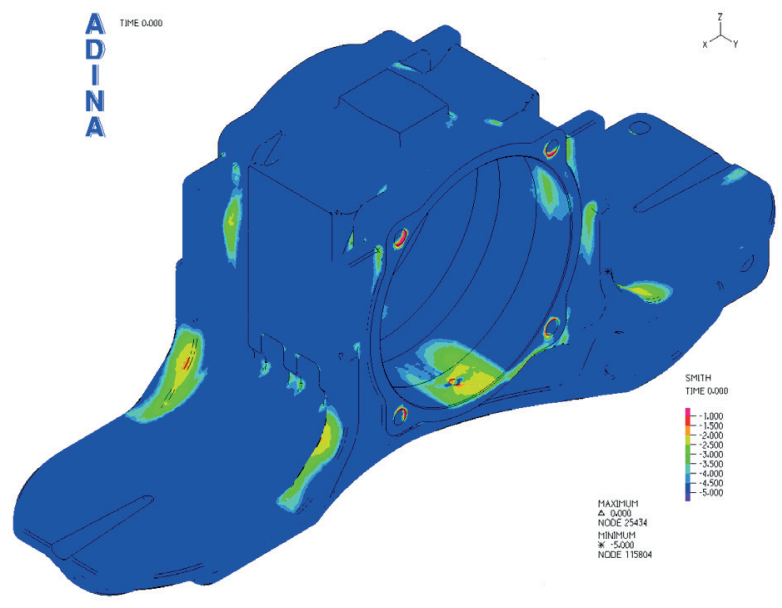

Fig. 7 Smith's safety factor

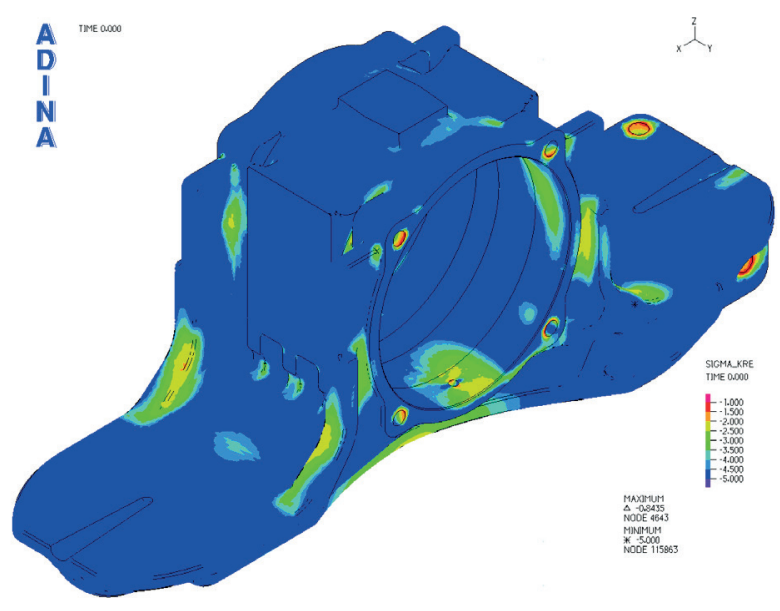

Fig. 8 Static safety factor 


\section{Conclusion}

Computer processing of the stress state analyses using FEM can significantly simplify and speed up the process. Manual processing of the results for the spectrum of load is difficult in terms of time and user. The above proposed algorithms process the results of the analysis to the cumulative form. The subsequent analysis of cumulative results becomes significantly simpler. The cumulative results are then displayed on a relatively small number of figures.

Another significant factor is that the results processing is independent of the user and can be performed in the user's absence. It is possible to process the results of analysis for the static spectrum of load of more than 1000 different modes during 24 hours. For the dynamic spectrum of load it is possible to process more than 350 different modes during 24 hours. In our presented example, one analysis of the stress state for a static or dynamic load mode (with considering the contact of bodies) takes approximately 4.5 hours.
Created algorithms, their implementation and use of powerful cluster computing resources allow efficient solving of the problems of the state of stress analysis or the life estimation of structures. Algorithms are applicable to the problems of contact (for static and dynamic spectrum of load) with a relatively large number of loading states in a short time (4-5 days including the processing of analyses results).

\section{Acknowledgements}

This contribution is the result of the project implementation: Development of optimum technology for the analysis of limit states of structural elements in contact, ITMS code 26220220118, supported by the Research \& Development Operational Programme funded by the ERDF.

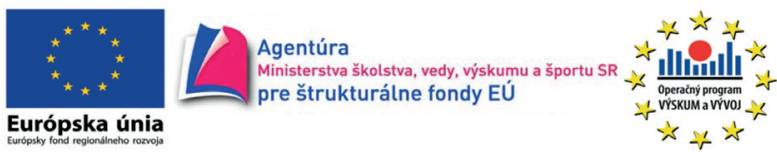

We support research activities in Slovakia /

The project is co-financed by the European Union.

\section{References}

[1] SAPIETOVA, A., SAPIETA, M., HYBEN, B.: Sensitivity Analysis Application for Multibody System Synthesis. Applied Mechanics and Materials, vol. 420, 2013, 68-73, ISSN 1660-9336.

[2] BATHE, K.J.: Finite Element Procedures. Prentice Hall, 1996.

[3] DEKYS, V., BRONCEK, J.: Measuring Strain of the Lattice Towers. Communications - Scientific Letters of the University of Zilina, vol. 14, No. 3, 2012, 39-42, ISSN 1335-4205.

[4] MURIN, J.: Finite Element Method for Truss and Frame Structures (in Slovak). Bratislava: STU Bratislava, 1999, 130 p., ISBN 80-227-1287-6.

[5] SAGA, M., KOPAS, P., VASKO, M.: Some Computational Aspects of Vehicle Shell Frames Optimization Subjected to Fatigue Life. Communications - Scientific Letters of the University of Zilina, vol. 12, No. 4, 2010, 73-79, ISSN 1335-4205.

[6] SAGA, M., VASKO, M., KOPAS, P.: Strength of Materials - Selected Methods and Applications (in Slovak). VTS: University of Zilina, 2011, 400 p., ISBN 978-80-89276-34-9.

[7] ZMINDAK, M., RIECKY, D.: Meshless Modelling of Laminate Mindlin Plates under Dynamic Loads. Communications - Scientific Letters of the University of Zilina, vol. 14, No. 3, 2012, 24-31, ISSN 1335-4205.

[8] ADINA - Theory and Modelling Guide, Volume 1 [help manual]. Watertown, 2010.

[9] RUZICKA, M.: Methods for the Fatigue Life Evaluating (in Czech). CVUT: Praha, 1999.

[10] KOPAS, P., SAGA, M., UHRICIK, M.: Contribution to Multiaxial Damage Calculation using FEM. Applied Mechanics and Materials, vol. 420, 2013, 318-224, ISSN 1660-9336.

[11] TREBUNA, F., BIGOS, P., JURICA, V., RITOK, J.: Possibility of Identifying the Residual Life of Machines and Equipment by Experimental Methods in Mechanics (in Slovak). Strojarstvo, Zilina, No. 2, 2000, 57-63.

[12] LEITNER, B.: Discrete optimization of the rail vehicle frame weight with respect to fatigue damage cumulation process. Transport means 2013 , proceedings of the $17^{\text {th }}$ international conference, October $24-25,2013$, Kaunas University of Technology, Lithuania, 17-20, ISSN 1822-296X.

[13] VASKO, A.: Analysis of the Factors Influencing Microstructure and Mechanical Properties of Austempered Ductile Iron. Communications - Scientific Letters of the University of Zilina, vol. 11, No. 4, 2009, 43-47, ISSN 1335-4205.

[14] BIGOS, P., KUBIN, K., KULKA, J., MANTIC, M., BURAK, J., PUSKAR, M., TANYASI, O., FALTINOVA, E.: Computational and Experimental Methods for Increasing Efficiency and Life Examination of Structures for Selected Power Drives and Transport Equipment (in Slovak). TU Kosice, 2010, 215 p., ISBN 978-80-553-0522-6. 


\section{COMMNICOIIONS}

[15] KOPAS, P., SAGA, M.: In-phase Multiaxial Fatigue Experimental Analysis of Welded Cylindrical 6063-T66 Aluminium Alloy Specimens. Manufacturing Technology, vol.13, No. 1, 2013, 59-64, ISSN 1213-2489.

[16] OCTAVE manual [help manual]. www.octave.org. 